\title{
ESTRUTURA DE DUAS FORMAÇÕES VEGETAIS DO CORDÃO EXTERNO DA RESTINGA DE MARAMBAIA, $\mathbf{R J}^{1}$
}

\author{
Luis Fernando Tavares de Menezes ${ }^{2}$ \\ Dorothy Sue Dunn de Araujo ${ }^{3}$
}

Recebido em 28/11/1997. Aceito em 25/07/1999

\begin{abstract}
RESUMO - (Estrutura de duas formações vegetais do cordão externo da restinga de Marambaia, RJ). Foram caracterizadas duas formações vegetais, psamófila-reptante e arbustiva de Palmae, no cordão arenoso externo da restinga de Marambaia (RJ) com base na posição topográfica, na fisionomia e na estrutura, utilizando-se o método de parcelas. Embora as duas formações sejam visualmente distintas, há uma zona de transição entre elas. Das 23 espécies amostradas na formação psamófila-reptante, Ipomoea pes-caprae, Ipomoea imperati, Allagoptera arenaria, Sporobolus virginicus, Remirea maritima e Panicum racemosum despontaram com maiores valores de importância (VI). A formação halófila dominada por Blutaparon portulacoides, típica de outras restingas no litoral brasileiro, não foi identificada devido ao intenso dinamismo da faixa entre o mar e a primeira linha de cristas praiais estabilizadas. A formação arbustiva de Palmae é dominada por Allagoptera arenaria, com 56\% do VI total, dentre as 64 espécies amostradas. As duas formações são dominadas por geófitas rizomatosas.
\end{abstract}

Palavras-chave - formações vegetais, fitossociologia, Marambaia, restinga

ABSTRACT - (Structure of two plant communities on Marambaia barrier island, Rio de Janeiro, Brazil).Two plant communities (creeping psamophyte and palm scrub) are described from the outer Marambaia beach ridge, Rio de Janeiro State, Brazil, based on topography, vegetation physiognomy, and structure. Although the two communities can be distinguished visually, they are joined by a transition zone. Ipomoea pes-caprae, Ipomoea imperati, Remirea maritima, Allagoptera arenaria, Sporobolus virginicus, and Panicum racemosum were the most important species sampled in the creeping psammophyte community out of a total of 23 . The halophyte community reported for other sandy coastal plains in Brazil was not observed at Marambaia due to the intense wave action on this beach. The palm scrub community was dominated by Allagoptera arenaria (56\% of total importance value) out of a total of 64 species. Rhizome-geophytes dominate both communities.

Key words - Brazil, phytosociology, plant communities, sandy coastal plain

\section{Introdução}

$\mathrm{Na}$ costa brasileira, as mais recentes classificações de formações vegetais em restingas foram feitas para o Pará (Bastos 1996), Espírito Santo (Pereira 1990), Rio de Janeiro (Araujo 1992), Paraná (Menezes-Silva 1998) e Rio Grande do Sul (Waechter 1990), sendo ainda necessárias

\footnotetext{
Parte da Dissertação de Mestrado do primeiro Autor

2 Departamento de Botânica, IB, Universidade Federal Rural do Rio de Janeiro, Antiga Rodovia Rio-São Paulo, Km 47, CEP 23.851-970, Seropédica, RJ, Brasil 3 Departamento de Ecologia, PPGE, Universidade Federal do Rio de Janeiro, CEP 21.941-570, Ilha do Fundão, Rio de Janeiro,
RJ, Brasil
} 
informações sobre a estrutura e a florística de muitos trechos do litoral para se chegar a uma classificação mais abrangente.

As formações halófila e psamófila-reptante, no sudeste brasileiro, foram reconhecidas por diversos autores, baseados na presença de espécies características e na posição relativa à praia (Araujo \& Henriques 1984; Pereira 1990; Araujo 1992; Lacerda et al. 1993). A halófila é localizada na parte superior da praia, de grande instabilidade de substrato, onde é lavada eventualmente pelas águas do mar, e a psamófila-reptante é localizada na faixa contígua (e mais alta) à halófila, atingida apenas pelas ondas nos períodos de tempestades. No litoral do Espírito Santo, por não existir distinção clara entre tais formações, foram tratadas por Thomaz \& Monteiro (1993) como uma só, a halófila-psamófila. Numa área de dunas ativas, na Praia do Peró (RJ), Cordeiro (1998) concluiu que, embora a distribuição das espécies obedeça a uma zonação paralela à praia, as espécies são substituídas de forma gradual, não sendo possível delimitar as duas formações. Contudo, Almeida \& Araujo (1997) reconheceram as duas formações na restinga de Massambaba, em Saquarema (RJ), baseados na cobertura percentual e na topografia. Estes autores também detectaram a presença de uma zona de transição entre estas duas formações. Informações para outras partes do litoral fluminense, com base nesses critérios de distinção entre estas formações, são ainda inexistentes.

Em muitas restingas do litoral sudeste, adjacente à formação psamófila-reptante e ainda no cordão externo de restinga, pode-se encontrar a formação Palmae (Pereira 1990) ou comunidade arbustiva de Palmae, assim denominada por Almeida \& Araujo (1997) por sua fisionomia homogênea, devido à dominância da palmeira-anã Allagoptera arenaria. Essa formação instala-se, muitas vezes, em locais onde anteriormente apresentava-se outro tipo de vegetação que tenha sido devastado, como a formação pós-praia ou a de Myrtaceae, por exemplo (Araujo 1992). Trabalhos recentes para o litoral do Espírito Santo (Cardoso 1995) e do Rio de Janeiro (Almeida \& Araujo 1997; Menezes 1996) apresentam dados sobre a estrutura desta formação, que está cada vez mais presente no litoral fluminense devido, muitas vezes, à devastação de vegetações mais fechadas.

A importância de novas investigações nessas formações vegetais deve-se à intensificação das atividades humanas nas restingas, que vêm sofrendo profundas alterações, principalmente nas comunidades vegetais próximas ao oceano. Esta faixa está sujeita às forças das marés mais altas e das ondas nos períodos de tempestades, e sua cobertura vegetal contribui de maneira significativa para a estabilidade destas areias, assim formando uma zona tampão entre o mar e as áreas interiores da planície. O conhecimento da estrutura destas formações fornecerá subsídios para manejo adequado das zonas de praia, principalmente em áreas altamente impactadas por atividades humanas.

Os objetivos deste estudo são os de caracterizar as formações vegetais que se desenvolvem entre a praia e a crista do cordão arenoso externo da restinga de Marambaia quanto à sua estrutura, posição topográfica e composição florística, bem como comparar estas formações com aquelas em posição equivalente em outros trechos do litoral sudeste/sul brasileiro.

\section{Material e métodos}

Área de estudo - a restinga de Marambaia localiza-se, em parte, na zona oeste do município do Rio de Janeiro e, em parte, nos municípios de Itaguaí e de Mangaratiba. Sua gênese foi descrita por Lamego (1946) e Roncarati \& Barrocas (1978), que postularam uma migração lateral da restinga na direção leste pela ação de correntes e afogamento dos vales fluviais durante a transgressão holocênica.

Atualmente, a restinga de Marambaia apresenta aproximadamente $40 \mathrm{~km}$ compr. no sentido W-E, emergindo poucos metros acima do nível médio da maré alta, exceto onde é coberta por dunas, algumas das quais com até $30 \mathrm{~m}$ alt. (Borges 1990). A extremidade da restinga, na ponta de Guaratiba, a leste, mede $1.800 \mathrm{~m}$ larg. (Borges 1990) e a extremidade que se apoia no Pico de Marambaia, a oeste, tem largura em torno de $3.500 \mathrm{~m}$. Neste trecho ocorre um sistema de dois 
cordões arenosos que vão se confluindo, formando um único cordão. Na parte central da restinga ocorre estreitamento de até $120 \mathrm{~m}$ larg., devido à remobilização dos sedimentos arenosos através das correntes internas da Baía de Sepetiba (Roncarati \& Barrocas 1978; Menezes et al. 1998).

A área escolhida para o desenvolvimento do presente estudo localiza-se na extremidade leste da restinga de Marambaia (aproximadamente $23^{\circ} 03^{\prime} \mathrm{S}$ e $44^{\circ} 03^{\prime} \mathrm{W}$ ). É constituída por uma faixa com $3 \mathrm{~km}$ de extensão e 70-90m larg., delimitada ao sul pelo mar e ao norte por uma estrada construída na crista do cordão externo. Esta faixa abrange, no perfil perpendicular ao mar, a anteduna e as cristas praiais originárias da ação das tempestades que ocasionam a elevação transitória do nível do mar; causando a erosão do prisma praial e sua posterior recomposição (Suguio \& Martin 1990). É possível distinguir visualmente duas fitofisionomias. A primeira é constituída por plantas herbáceas estoloníferas, rizomatosas e reptantes e localiza-se sobre a anteduna; as cristas praiais e as depressões entre estas são recobertas por vegetação fisionomicamente distinta da primeira, constituída principalmente por espécies herbáceas e subarbustivas de porte baixo (altura média $=0,5 \mathrm{~m}$ ), dominada fisionomicamente pela palmeira Allagoptera arenaria. Devido às manobras militares na área, a última está sujeita a freqüentes queimadas.

Os dados climáticos (procedentes da estação meteorológica de Marambaia, distante $500 \mathrm{~m}$ da área de estudo, e cedidos pelo Instituto Nacional de Meteorologia - INMET) correspondem ao período de 1984 a 1995 (12 anos). A temperatura média anual é de $23,6^{\circ} \mathrm{C}$, sendo fevereiro o mês mais quente, com temperatura média de $26,7^{\circ} \mathrm{C}$ e julho, o mais frio, com temperatura média de $21,0^{\circ} \mathrm{C}$. Não há estação seca durante o ano. O total médio anual de precipitação é de $1.027 \mathrm{~mm}$, sendo agosto o mês mais seco, com média de $47,4 \mathrm{~mm}$, e março, o mais chuvoso, com média de $140,6 \mathrm{~mm}$.

Metodologia - foram elaborados seis perfis topográficos na área de estudo utilizando-se o método de Garcia \& Piedade (1987), com adaptações. Com o auxílio de bússola e trena, determinou-se uma linha reta e, ao longo desta, estabeleceram-se as diferenças de níveis com o auxílio de régua de carpinteiro e clinômetro (marca Suunto, modelo PM-5/360PC). O ponto zero, determinado na área desnuda da praia acima da zona de estirâncio, foi apenas uma referência para os perfis, não tendo relação com o nível do mar. Cada perfil topográfico é representativo de uma faixa longitudinal homogênea da praia, onde foram localizadas de uma a três linhas de amostragem.

A movimentação de areia pelo vento (acúmulo e erosão) na área vegetada foi estimada através de observações mensais de três estacas de madeira, graduadas até $30 \mathrm{~cm}$ acima e abaixo do nível do solo, no período de outubro/1994 a setembro/1995. As estacas foram dispostas em três pontos ao longo da praia (distantes $400 \mathrm{~m}$ entre si), perpendiculares à linha da praia e distantes umas das outras em cerca de $30 \mathrm{~m}$. A primeira estaca de cada grupo foi fixada no ponto onde, aparentemente, não havia mais influência das ondas; a segunda, no cume da primeira linha de cristas praiais, fixadas predominantemente por indivíduos de Allagoptera arenaria e, a última, na depressão entre as duas últimas linhas de cristas praiais.

Para a análise estrutural da vegetação utilizou-se parcelas de $1 \mathrm{~m}^{2}$, dimensão adequada ao tamanho e espaçamento dos indivíduos em toda a área de estudo, i.e., nas duas fitofisionomias (Mueller-Dombois \& Ellenberg 1974). As parcelas foram distribuídas em intervalos de $1 \mathrm{~m}$ ao longo de 13 linhas orientadas perpendicularmente à linha da praia. O comprimento das linhas variou conforme a distância entre a praia e a crista do cordão externo, perfazendo o total de $544 \mathrm{~m}^{2}$ amostrados.

Em cada parcela foi estimada a cobertura de copa de cada espécie (área ocupada pela projeção da parte aérea da planta, estimada como percentagem e expressa em $\mathrm{m}^{2}$ - Brower \& Zar 1977). Os valores relativos de dominância (cobertura) e freqüência foram utilizados para determinar o valor de importância (VI). A densidade não foi determinada devido à dificuldade de individualização das plantas das espécies mais freqüentes (muitas vezes estoloníferas ou rizomatosas), inclusive Allagoptera arenaria (Leite 
1990). Segundo Mueller-Dombois \& Ellenberg (1974), qualquer um dos três parâmetros densidade, dominância, freqüência - pode ser interpretado como um "VI", já que é o investigador quem determina qual destes é o mais importante para alcançar os objetivos da pesquisa.

Os mesmos autores consideram que, para análise descritiva de formações herbáceas ou baixo-arbustivas, estimativas de cobertura e freqüência são suficientes, sendo desnecessário o esforço demandado na determinação da densidade. Este procedimento já foi seguido em outros trabalhos com vegetação da praia (Thomaz 1991; Pereira et al. 1992; Almeida \& Araujo 1997). É também comum encontrar definições diversas para o VI em trabalhos realizados recentemente em outros tipos de vegetação, com diversos objetivos. Por exemplo, este valor é definido como a soma dos valores relativos de área basal e densidade em estudo de recuperação de floresta após distúrbio, em Porto Rico (Frangi \& Lugo 1998); como a média dos valores relativos de densidade e freqüência em estudo de plântulas e jovens em floresta em regeneração, após corte seletivo na Argentina (Montagnini et al. 1998); e como a média dos valores relativos de densidade e volume num estudo das relações entre distribuição de espécies e fatores abióticos numa floresta seca no Brasil central (Oliveira-Filho et al. 1998).

A separação das formações psamófilareptante e arbustiva de Palmae para fins de análise estrutural foi estabelecida visualmente na zona de transição entre as duas formações, isto é, onde a cobertura de copa de Allagoptera arenaria sofre aumento abrupto.

Na confecção das curvas do coletor, elaboradas para cada formação (psamófila-reptante e arbustiva de Palmae), verificou-se, primeiramente, a área total compreendida pelas parcelas da primeira linha amostrada (perpendicular à praia) e o número de espécies contidas na mesma. $\mathrm{O}$ surgimento de novos táxons foi estabelecido a partir do acréscimo da área das linhas subseqüentes.

Para as espécies vegetais identificadas na área de estudo adotou-se o critério de classificação quanto à forma de vida apresentado por MuellerDombois \& Ellenberg (1974). O material botânico coletado foi depositado no Herbário da Universidade Federal Rural do Rio de Janeiro (RBR).

\section{Resultados}

Os perfis topográficos, com 110 a $129 \mathrm{~m}$ compr. apresentaram microrrelevo bastante irregular, com 4- 6 cristas praiais separadas por pequenas depressões (Fig. 1). A altura máxima das cristas em relação ao ponto 0 variou de 2 a $4 \mathrm{~m}$. Não houve movimentação da areia pelo vento durante o período dos trabalhos de campo.

A formação psamófila-reptante encontra-se na zona contígua à areia desnuda da praia, ocupando faixa de 17 a $36 \mathrm{~m}$ larg. (Fig. 1). Recobre sempre a primeira crista do perfil, às vezes estendendo-se até a segunda crista; o limite interno não parece estar relacionado à topografia.

As espécies mais freqüentes na formação psamófila-reptante nos seis perfis topográficos são Ipomoea imperati, Ipomoea pes-caprae, Remirea maritima, Panicum racemosum e Sporobolus virginicus. As quatro primeiras espécies ocorrem em todos os perfis, porém $S$. virginicus está ausente do perfil 5. A presença de Allagoptera arenaria na formação psamófila-reptante junto com algumas outras espécies de maior freqüência em áreas mais distantes da praia (Cereus fernambucensis, Chamaecrista flexuosa, Setaria setosa, Vernonia scorpioides) caracteriza a presença de zona de transição entre as duas formações visualmente distintas. $R$. maritima, $I$. imperati e $P$. racemosum também são encontradas na faixa dominada por A. arenaria.

Blutaparon portulacoides e Scaevola plumieri estão presentes somente na formação psamófila-reptante. A primeira espécie aparece em dois perfis, ora no início desta formação (perfil 1), ora contígua à formação arbustiva de Palmae (perfil 4). S. plumieri é encontrada no início ou no meio da faixa da formação psamófila-reptante.

O total de 23 espécies foi amostrado na formação psamófila-reptante (Tab. 1), ocorrendo estabilização na curva de coletor em $100 \mathrm{~m}^{2}$ (Fig. 2). As espécies que despontaram com maior VI foram: Ipomoea pes-capre, Ipomoea imperati, Remirea maritima, Allagoptera arenaria, Sporo- 

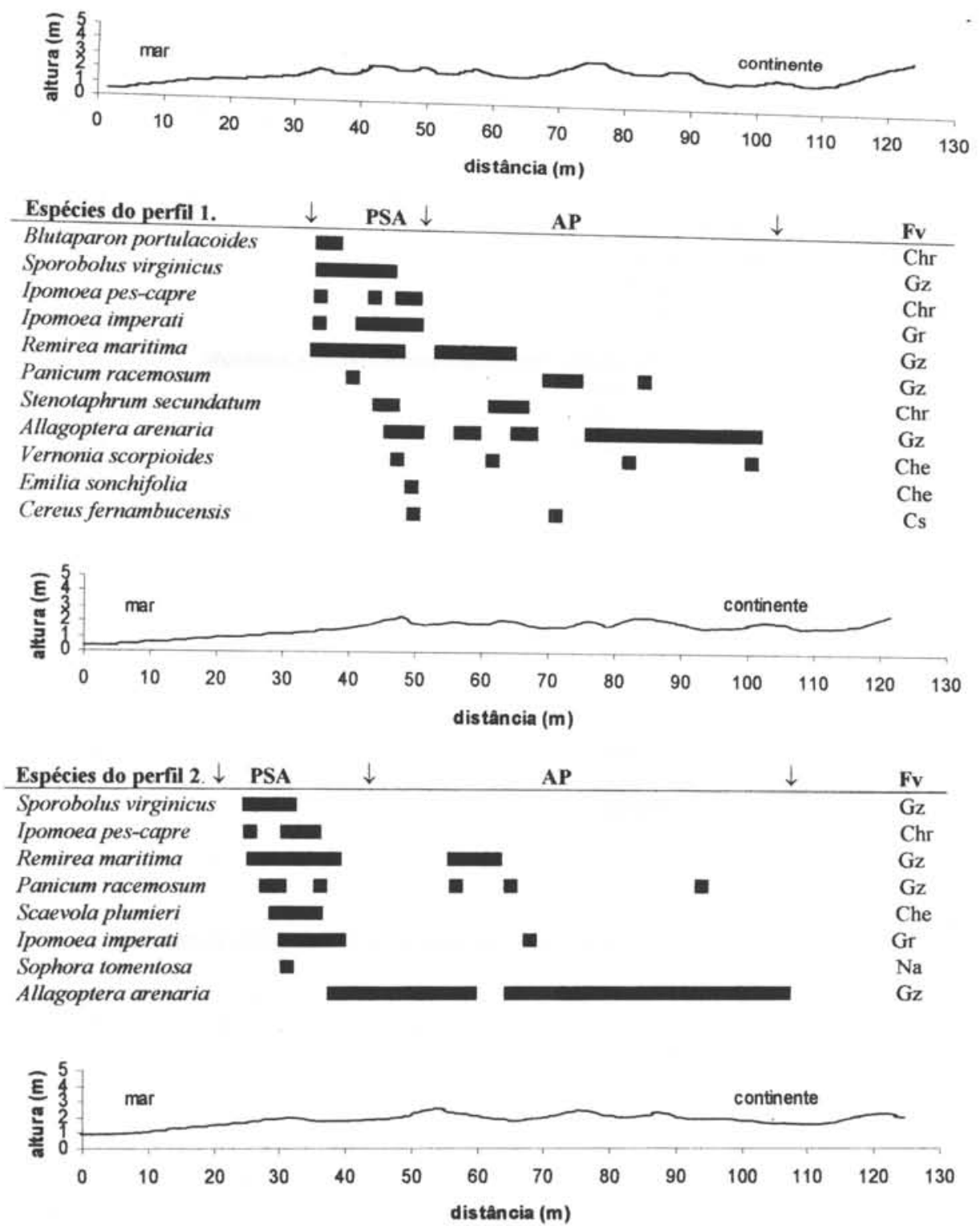

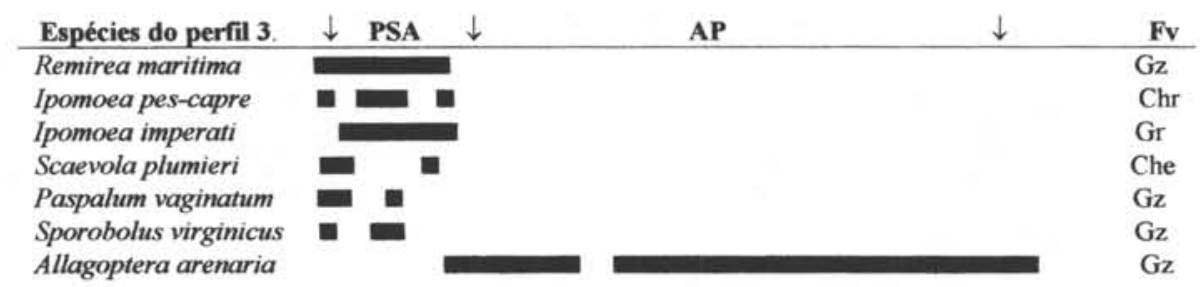

Figura 1. Ocorrência das espécies da comunidade psamófila-reptante (PSA) ao longo de cinco perfis topográficos, no cordão externo da restinga de Marambaia, RJ. As setas indicam os limites de distribuição destas espécies na formação psamófila-reptante e na arbustiva de Palmae (AP). As formas de vidas (FV) estão indicadas por: Geófita rizomatosa (Gz), Nanofanerófita (Na), Caméfita herbácea escaposa (Che), Trepadeira geofítica (Tg), Caméfita suculenta (Cs), Geófita radicigema (Gr), Hemicriptófita escaposa rosetada
(Her), Caméfita herbácea reptante (Chr). 

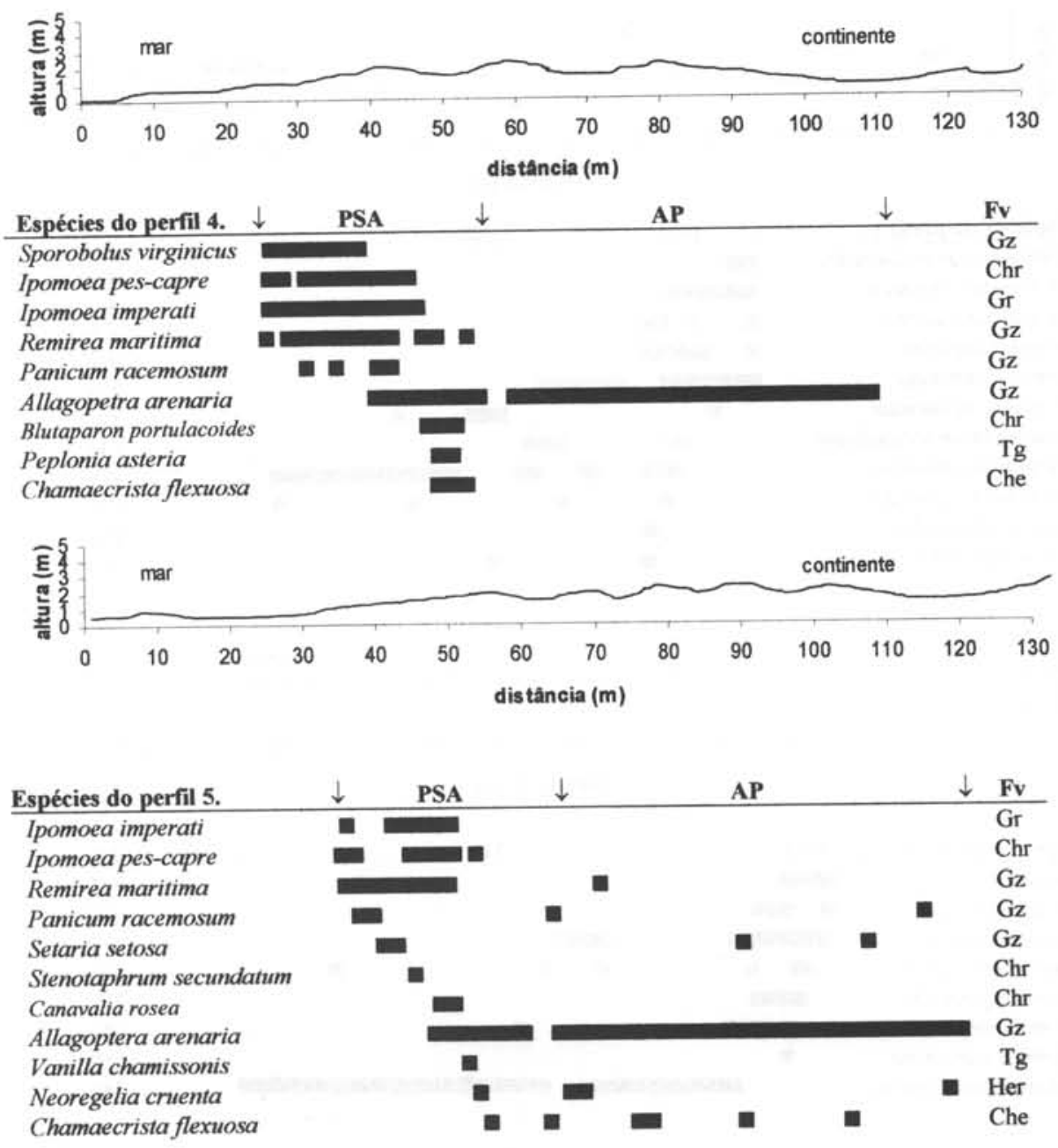

Figura 1. continuaçāo

bolus virginicus e Panicum racemosum, englobando $84 \%$ do total de VI (Tab. 2). A presença de A. arenaria neste grupo de espécies é mais uma indicação de que a passagem de uma formação para outra é gradual. Entretanto, a cobertura total desta espécie é apenas $4 \%$ da cobertura total na formação arbustiva de Palmae.

A formação arbustiva de Palmae apresentou largura média de $65 \mathrm{~m}$. A espécie dominante, $A$. arenaria $(56 \%$ do VI total - Tab. 3) está presente em praticamente toda a extensão do perfil. A curva do coletor para essa formação não apresentou tendência à estabilização na área de $403 \mathrm{~m}^{2}$ de amostragem (Fig. 2b). Essa formação apresentou o total de 64 táxons (Tab. 1).

As duas formações apresentam diferenças nítidas nas formas de vida dominantes (Tab. 4). $\mathrm{Na}$ psamófila-reptante, as geófitas rizomatosas correspondem à metade do VI total, sendo que $R$. maritima, A. arenaria, $S$. virginicus e $P$. racemosum são as espécies mais importantes neste grupo. As geófitas radicigemas (principalmente I. imperati) também são importantes nesta formação. Na formação arbustiva de Palmae, as geófitas rizomatosas foram responsáveis por $61 \%$ do VI total. Considerando esse percentual, $90 \%$ 

Tabela 1. Relação das espécies amostradas e observadas nas formações psamófila-reptante (PSA) e arbustiva de Palmae (AP), na
restinga de Marambaia, RJ.

\begin{tabular}{|c|c|c|c|}
\hline Espécies & Familias & PSA & AP \\
\hline Blutaparon portulacoides (A. St. Hil.) Mears & Amaranthaceae & $X$ & \\
\hline Alternanthera littoralis (Mart.) A. St.-Hil. & Amaranthaceae & $\mathrm{X}$ & \\
\hline Schinus terebinthifolius Raddi & Anacardiaceae & & $\mathrm{X}$ \\
\hline Hydrocotyle bonarienesis Lam. & Apiaceae & $\mathrm{X}$ & $\mathrm{X}$ \\
\hline Allagoptera arenaria (Gomes) Kuntze & Arecaceae & $x$ & $\mathrm{X}$ \\
\hline Matelea maritima (Jacq.) Woodson & Asclepiadaceae & & $\mathrm{X}$ \\
\hline Peplonia asteria (Vell.) Fontella \& E.A.Schwarz & Asclepiadaceae & $\mathrm{X}$ & $x$ \\
\hline Oxypetalum banksii Schult. spp. banksii & Asclepiadaceae & & $X$ \\
\hline Mikania stipulacea (M.Vahl) Willd. & Asteraceae & & $x$ \\
\hline Asteraceae & Asteraceae & & $x$ \\
\hline Emilia sonchifolia $\mathrm{L}$. & Asteraceae & $X$ & $x$ \\
\hline Vernonia scorpioides (Lam.) Pers. & Asteraceae & $X$ & $x$ \\
\hline Pseudobombax grandiflorum (Cav.) A. Robyns & Bombacaceae & & $\mathrm{X}$ \\
\hline Tournefortia villosa Salzm. & Boraginaceae & & $x$ \\
\hline Neoregelia cruenta (Graham) L. B. Sm. & Bromeliaceae & $\mathrm{X}$ & $x$ \\
\hline Quesnelia quesneliana (Brongn.) L. B. Sm. & Bromeliaceae & & $x$ \\
\hline Bromelia antiacantha Bertol. & Bromeliaceae & & $\mathrm{x}$ \\
\hline Pilosocereus arrabidae (Lem.) Byles \& G. D. Rowley & Cactaceae & & $\mathrm{X}$ \\
\hline Cereus fernambucensis Lem. & Cactaceae & $\mathrm{X}$ & $\mathrm{x}$ \\
\hline Chamaecrista ramosa (Vogel) H. S. Irwin \& R. C. Barneby & Caesalpiniaceae & & $\mathrm{X}$ \\
\hline C. flexuosa (L.) Greene & Caesalpiniaceae & $\mathrm{X}$ & $\mathrm{x}$ \\
\hline Acicarpha spatulata $\mathrm{R}$. Br. & Calyceraceae & $\mathrm{x}$ & \\
\hline Ipomoea imperati (Vahl.) Griseb. & Convolvulaceae & $\mathrm{X}$ & $\mathrm{X}$ \\
\hline I. pes-capre (L.) Sweet. & Convolvulaceae & $\mathrm{x}$ & $\mathrm{x}$ \\
\hline Jacquemontia holosericea (Weinm.) O'Donell & Convovulaceae & & $x$ \\
\hline Remirea maritima Aubl. & Cyperaceae & $\mathrm{X}$ & $\mathrm{x}$ \\
\hline Erythroxylum ovalfolium Pey. & Erythroxylaceae & & $x$ \\
\hline Chaetocarpus myrsinites Baill. & Euphorbiaceae & & $\mathrm{X}$ \\
\hline Chamaecyse thymifolia (L.) Millsp. & Euphorbiaceae & $\mathrm{X}$ & $\mathrm{X}$ \\
\hline Sebastiana curniculata (Vahl) Muell. Arg. & Euphorbiaceae & & $\mathrm{X}$ \\
\hline Vigna peduncularis Fawc. \& Rendle & Fabaceae & & $\mathrm{X}$ \\
\hline Canavalia rosea (Sw.) DC. & Fabaceae & $\mathrm{X}$ & $\mathrm{X}$ \\
\hline Centrosema virginianum (L.) Benth. & Fabaceae & & $\mathrm{X}$ \\
\hline Clitoria sp. & Fabaceae & $X$ & $\mathrm{X}$ \\
\hline Desmodium barbatum (L.) Benth. & Fabaceae & & $\mathrm{X}$ \\
\hline Stylosanthes viscosa Sw. & Fabaceae & & $\mathrm{X}$ \\
\hline Sophora tomentosa spp. litoralis (Schrad.) Yakovl. & Fabaceae & $\mathrm{X}$ & $\mathrm{X}$ \\
\hline Scaevola plumieri (L.) Vahl. & Goodeniaceae & $\mathrm{X}$ & \\
\hline Byrsonima sericea DC. & Malpighiaceae & & $\mathrm{x}$ \\
\hline Heteropterys chrysophylla (Lam.) Kunth. & Malpighiaceae & & $\mathrm{X}$ \\
\hline Stigmaphyllon paralias A. Juss. & Malpighiaceae & & $\mathrm{X}$ \\
\hline Norantea brasiliensis Choisy & Marcgraviaceae & & $\mathrm{X}$ \\
\hline Tibouchina sp. & Melastomataceae & & $\mathrm{x}$ \\
\hline Inga maritima Benth. & Mimosaceae & & $\mathrm{X}$ \\
\hline Eugenia nitida Cambess. & Myrtaceae & & $\mathrm{x}$ \\
\hline E. ovalifolia Cambess. & Myrtaceae & & $\mathrm{x}$ \\
\hline E. rotundifolia Casar. & Myrtaceae & & $\mathrm{X}$ \\
\hline Myrrhinium atropurpureum Schott & Myrtaceae & & $\mathrm{X}$ \\
\hline Guapira opposita (Vell.) Reitz & Nyctaginaceae & & $\mathrm{X}$ \\
\hline Ouratea cuspidata (St. Hil.) Engl. & Ochnaceae & & $\mathrm{X}$ \\
\hline Cyrtopodium polyphyllum (Vell.) Pabst ex F. Barros & Orchidaceae & & $x$ \\
\hline Epidendrum denticulatum Barb. Rodr. & Orchidaceae & & $\mathrm{X}$ \\
\hline Aanilla chamissonis var. brevifolia Cogn. & Orchidaceae & $\mathrm{X}$ & $\mathrm{X}$ \\
\hline Catasetum sp. & Orchidaceae & & $\mathrm{x}$ \\
\hline
\end{tabular}




\begin{tabular}{|c|c|c|c|}
\hline Espécies & Famílias & PSA & AP \\
\hline Panicum racemosum (P. Beauv.) Spreng. & Poaceae & $\mathrm{X}$ & $\mathrm{X}$ \\
\hline Setaria setosa (Swartz) P. Beauv. & Poaceae & $\mathrm{X}$ & $\mathrm{X}$ \\
\hline Stenotaphrum secundatum (Walter) Kuntze & Poaceae & $\mathrm{X}$ & $X$ \\
\hline Paspalum arenarium Schrad. & Poaceae & & $\mathrm{X}$ \\
\hline$P$. vaginatum $\mathrm{Sw}$. & Poaceae & $\mathrm{X}$ & \\
\hline Sporobolus virginicus (L.) Kunth & Poaceae & $\mathrm{X}$ & $\mathrm{X}$ \\
\hline Polygala cyparissias A. St.-Hil. & Polygonaceae & $\mathrm{X}$ & \\
\hline Portulaca mucronata Link & Portulacaceae & & $\mathrm{X}$ \\
\hline Borreria brachysteonoides Cham. \& Schltdl. & Rubiaceae & & $\mathrm{X}$ \\
\hline B. verticillata (L.) Meyer & Rubiaceae & & $\mathrm{X}$ \\
\hline Diodea radula (Willd. \& Hoffmanns.) Cham. \& Schltdl. & Rubiaceae & & $\mathrm{X}$ \\
\hline Tocoyena bullata Mart. & Rubiaceae & & $\mathrm{X}$ \\
\hline Diodea conferta $($ Schott) DC. & Rubiaceae & & $\mathrm{X}$ \\
\hline Paullinea coriacea Casar. & Sapindaceae & & $\mathrm{X}$ \\
\hline Manilkara subsericea (Mart.) Dubard & Sapotaceae & & $\mathrm{X}$ \\
\hline Smilax rufescens Griseb. & Smilaceae & & $\mathrm{X}$ \\
\hline
\end{tabular}

foi devido à A. arenaria. As nanofanerófitas (e.g., Inga maritima) e caméfitas herbáceas escaposas (e.g., Stylosanthes viscosa, Chamaecrista flexuosa) apresentaram VI bem maiores nessa formação, cada grupo correspondendo a aproximadamente $12 \%$ do VI total.

\section{Discussão}

A praia da restinga de Marambaia é área de intenso dinamismo. A suavidade de perfil da praia possibilita que a formação psamófila-reptante seja atingida com freqüência pelo mar. A ação das vagas na linha de costa propicia mudanças bastante profundas, que influenciam diretamente a estrutura desta formação e também a sua largura. A ocorrência de Scaevola plumieri no início da formação psamófila-reptante, contígua à areia desnuda da praia, e não nas partes mais estabilizadas do cordão externo, onde geralmente se localiza (Araujo \& Oliveira 1988), comprova que o perfil da praia é modificado pela erosão e subsequente sedimentação durante o ciclo mensal das marés (Suguio \& Martin 1990). Da mesma forma, a presença de Blutaparon portulacoides, espécie tipicamente encontrada na faixa contígua à areia
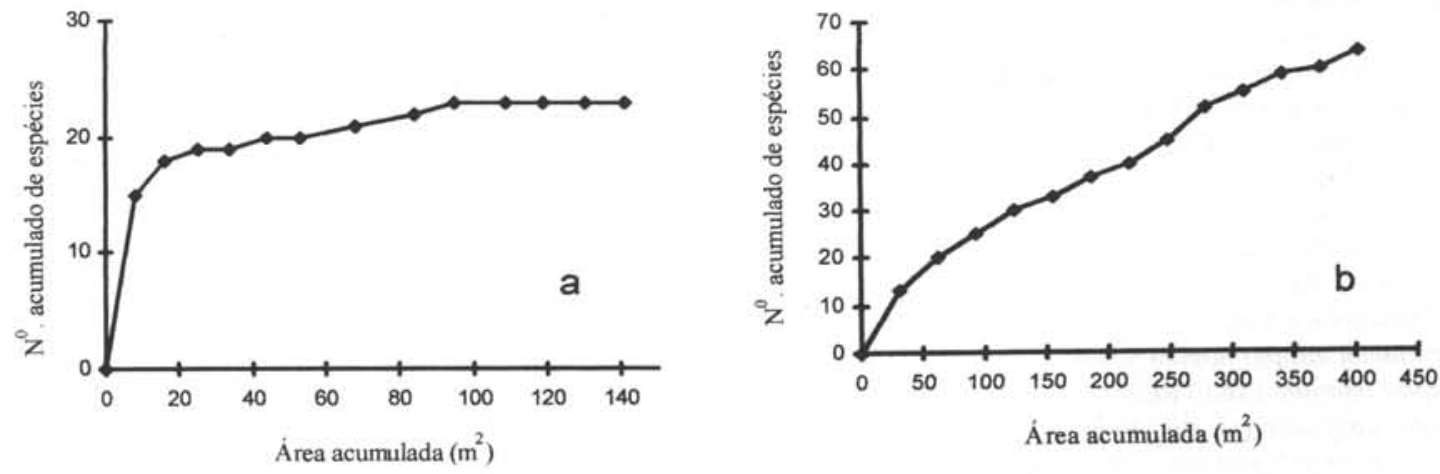

Figura 2. Curva do coletor para a formação psamófila-reptante (a), com o total de 141 parcelas, e para a formação arbustiva Palmae (b), com o total de 403 parcelas, no cordão externo da restinga de Marambaia, RJ. 
Tabela 2. Parâmetros fitossociológicos das espécies com valor de importância (VIt) $>1$ da formação psamófila-reptante da restinga de Marambaia, $\mathrm{RJ} . \mathrm{NPe}=$ Número de parcelas onde se encontra a espécie; $\mathrm{CT}=$ Cobertura total da espécie $\left(\mathrm{m}^{2}\right) ; \mathrm{FR} t=$ Frequêencia relativa do táxon (\%); DoRt= Dominância relativa do táxon (\%); VIt = Valor de importância do táxon (\%).

\begin{tabular}{lrrrrr}
\hline Espécies & NPe & CT & FRt* & DoRt* & Vit** \\
\hline Ipomoea pes-caprae & 83 & 706 & 15,3 & 21,7 & 37,1 \\
I. imperati & 113 & 483 & 20,9 & 14,9 & 35,8 \\
Remirea maritima & 115 & 448 & 21,3 & 13,8 & 35,0 \\
Allagoptera arenaria & 29 & 775 & 5,4 & 23,9 & 29,2 \\
Sporobulus virginicus & 77 & 195 & 14,2 & 6,0 & 20,2 \\
Panicum racemosum & 44 & 95 & 8,1 & 2,9 & 11,1 \\
Scaevola plumieri & 8 & 200 & 1,5 & 6,2 & 7,6 \\
Blutaparon portulacoides & 17 & 131 & 3,1 & 4,0 & 7,2 \\
Stenotaphrum secundatum & 18 & 60 & 3,3 & 1,8 & 5,2 \\
Paspalum vaginatum & 16 & 34 & 3,0 & 1,0 & 4,0 \\
Canavalia rosea & 5 & 31 & 0,9 & 1,0 & 1,9 \\
Neoregelia cruenta & 1 & 40 & 0,2 & 1,2 & 1,4 \\
\hline * E & & & &
\end{tabular}

* $\Sigma$ das 23 espécies $=100 \% * * \sum$ das 23 espécies $=200 \%$

Tabela 3. Parâmetros fitossociológicos das espécies com valor de importância (VIt) $>1$ da formação arbustiva de Palmae da restinga de Marambaia, RJ. $\mathrm{NPe}=$ Número de parcelas onde está a espécie; $\mathrm{CT}=$ Cobertura total da espécie $\left(\mathrm{m}^{2}\right) ; \mathrm{FRt}=$ Freqüência relativa do táxon (\%); DoRt = Dominância relativa do táxon (\%); VIt = Valor de importância do táxon (\%).

\begin{tabular}{|c|c|c|c|c|c|}
\hline Espécies & $\mathrm{NPe}$ & $\mathrm{CT}$ & FRt* & DoRt* & Vit** \\
\hline Allagoptera arenaria & 364 & 19691 & 32,1 & 78,9 & 111,0 \\
\hline Inga maritima & 65 & 1005 & 5,7 & 4,0 & 9,8 \\
\hline Manilkara subsericea & 23 & 591 & 2,0 & 2,4 & 4,4 \\
\hline Stylosanthes viscosa & 43 & 138 & 3,8 & 0,6 & 4,3 \\
\hline Setaria setosa & 44 & 74 & 3,9 & 0,3 & 4,2 \\
\hline Cereus fernambucensis & 37 & 205 & 3,3 & 0,8 & 4,1 \\
\hline Chamaecrista flexuosa & 39 & 95 & 3,4 & 0,4 & 3,8 \\
\hline Desmodium barbatum & 37 & 85 & 3,3 & 0,3 & 3,6 \\
\hline Panicum racemosum & 35 & 69 & 3,1 & 0,3 & 3,4 \\
\hline Vernonia scorpioides & 31 & 103 & 2,7 & 0,4 & 3,1 \\
\hline Stigmaphyllon paralias & 29 & 140 & 2,6 & 0,6 & 3,1 \\
\hline Pilosocereus arrabidae & 30 & 90 & 2,6 & 0,4 & 3,0 \\
\hline Eugenia nitida & 19 & 307 & 1,7 & 1,2 & 2,9 \\
\hline Vanilla chamissonis & 26 & 148 & 2,3 & 0,6 & 2,9 \\
\hline Neoregelia cruenta & 14 & 340 & 1,2 & 1,4 & 2,6 \\
\hline Clitoria sp. & 23 & 89 & 2,0 & 0,4 & 2,4 \\
\hline Remirea maritima & 24 & 56 & 2,1 & 0,2 & 2,3 \\
\hline Diodea conferta & 20 & 51 & 1,8 & 0,2 & 2,0 \\
\hline Borreria brachysteonoides & 20 & 40 & 1,8 & 0,2 & 1,9 \\
\hline Centrosema virginianum & 18 & 58 & 1,6 & 0,2 & 1,8 \\
\hline Diodea radula & 17 & 52 & 1,5 & 0,2 & 1,7 \\
\hline Hydrocotyle bonariensis & 17 & 30 & 1,5 & 0,1 & 1,6 \\
\hline Paspalum arenarium & 15 & 66 & 1,3 & 0,3 & 1,6 \\
\hline Ouratea cuspidata & 6 & 185 & 0,5 & 0,7 & 1,3 \\
\hline Jacquemontia holosericea & 9 & 81 & 0,8 & 0,3 & 1,1 \\
\hline Erythroxylum ovalifolium & 3 & 210 & 0,3 & 0,8 & 1,1 \\
\hline Myrrhinium atropurpureum & 7 & 111 & 0,6 & 0,4 & 1,1 \\
\hline Paullinea coriacea & 9 & 58 & 0,8 & 0,2 & 1,0 \\
\hline Epidendrum denticulatum & 9 & 57 & 0,8 & 0,2 & 1,0 \\
\hline
\end{tabular}

* $\sum$ das 64 espécies $=100 \% * * \sum$ das 64 espécies $=200 \%$ 
Tabela 4. Número de espécies em cada categoria de forma de vida e o seu respectivo valor de importância (VI= frequiência relativa + dominância relativa) nas formaçōes vegetais psamófila-reptante e arbustiva de Palmae , no cordão externo da restinga de Marambaia, RJ. As formas de vida seguem a classificação de Mueller-Dombois \& Ellenberg (1974).

\begin{tabular}{lcccc}
\hline Forma de vida & \multicolumn{2}{c}{ Psamófila-reptante } & \multicolumn{2}{c}{ Arbustiva de Palmae } \\
\cline { 2 - 5 } & Número de espécies & VI total $(\%)^{*}$ & Número de espécies & VI total $(\%)^{*}$ \\
\hline Geófita rizomatosa & 6 & 100,2 & 6 & 122,8 \\
Nanofanerófita & 1 & 0,2 & 16 & 24,0 \\
Caméfita herbácea escaposa & 6 & 10,0 & 16 & 23,8 \\
Trepadeira geofítica & 2 & 0,6 & 9 & 8,3 \\
Caméfita herbácea pulvinada & - & - & 1 & 4,3 \\
Caméfita suculenta & 1 & -2 & 1 & 4,1 \\
Fanerófita suculenta & - & 36,0 & 4 & 3,0 \\
Geófita radicigema & 2 & 1,4 & 3 & 2,8 \\
Hemicriptófita escaposa rosetada & 1 & - & 3 & 3,2 \\
Liana fanerófita & - & 51,3 & 3 & 1,5 \\
Caméfita herbácea reptante & 4 & - & 1 & 1,4 \\
Epífita & - & & 1,0
\end{tabular}

* $\Sigma$ de todas as formas de vida $=200 \%$

desnuda da praia, ocorrendo junto com os primeiros indivíduos de $A$. arenaria, é indício deste dinamismo. Esta distribuição espacial de $B$. portulacoides foi observada por Pereira et al. (1992) no Espírito Santo e relacionada à ocorrência de cristas de praia formadas em épocas diferentes, provavelmente por ondas de tempestades.

Na restinga de Marambaia, a presença de $B$. portulacoides em ambos os limites da formação psamófila-reptante (interno e externo) e sua ausência total em outros trechos não permitiu a delimitação da formação halófila e sua distinção da psamófila-reptante, como tem sido reportado para outros trechos do litoral sudeste brasileiro (Pereira et al. 1992; Almeida \& Araujo 1997). Esta formação é definida basicamente por sua posição topográfica e composição florística, sendo dominado por B. portulacoides. Por exemplo, na região de Saquarema (Almeida \& Araujo 1997), esta espécie mostrava nítida diminuição na cobertura, da praia para o interior da planície. Os perfis topográficos em Saquarema são bem distintos dos perfis em Marambaia e, aparentemente, a presença de desnível mais abrupto na zona onde começa a cobertura vegetal, contígua à praia, resultado de acentuada erosão, define com mais precisão a área de ocorrência de $B$. portulacoides. Deve ser lembrado, entretanto, que o perfil topográfico desta zona altera-se com muita freqüência e, por isso, seria necessário acompanhamento da cobertura vegetal ao longo de vários períodos de tempestades e calmarias, para se entender a dinâmica destes processos.

$\mathrm{Na}$ zona de contato entre as duas formações vegetais do cordão externo em Marambaia, fisionômica e estruturalmente distintas, há certo grau de sobreposição de espécies, constituindo zona de transição. Esta mudança gradual de uma formação para outra era esperada devido à própria natureza do ambiente das restingas, onde os cordões arenosos são depositados paralelamente à linha da praia, e diversos fatores ambientais variam ao longo de um gradiente na direção marcontinente (e.g., intensidade do vento, freqüência de inundação, estabilidade do substrato, deposição de salsugem, conteúdo de nutrientes no solo Araujo 1992; Seeliger 1992; Lacerda et al. 1993). Zonas de transição entre diferentes tipos de vegetação nas planícies arenosas já foram relatadas para diversas regiões do litoral brasileiro (Pfadenhauer 1993; Almeida \& Araujo 1997; Pereira et al. 1992, Oliveira-Filho 1993).

Formação psamófila-reptante - a estabilização da curva do coletor na formação psamófilareptante indica que a flora foi bem representada na amostragem. Devido à baixa riqueza desta formação ( 23 espécies) e ao número relativamente elevado de unidades de amostragem (141 par- 
celas), a existência de uma zona de transição entre a formação psamófila-reptante e a arbustiva de Palmae não influenciou a estabilização da curva do coletor. As três espécies que foram observadas nesta formação, mas que não fizeram parte das amostragens (Alternanthera littoralis, Acicarpha spatulata e Polygala cyparissias) são pouco comuns no local.

A composição florística da formação psamófila-reptante foi semelhante a de outros trechos do litoral sul e sudeste brasileiro. Na descrição dos tipos de vegetação da restinga de Carapebus (RJ), Henriques et al. (1986) apontam Blutaparon portulacoides, juntamente com Panicum racemosum e Sporobolus virginicus como as espécies mais abundantes na zona praiana. Em estudos anteriores realizados em formações vegetais de praias (Dansereau 1947; Magnanini 1954; Hueck 1955; Pfadenhauer 1978; Hay et al. 1981), B. portulacoides também é apontada como sendo uma das mais abundantes. Contudo, a frequiência relativa desta espécie em Marambaia foi baixa $(3,14 \%)$. Em outros estudos quantitativos realizados no litoral do Estado do Rio de Janeiro, também se verificou freqüência relativa baixa para esta espécie (Henriques et al. 1984; Almeida \& Araujo 1997). O mesmo foi observado para o litoral do Espírito Santo, estando até mesmo ausente em algumas áreas (Thomaz 1991).

Henriques et al. (1984) atribuíram a diminuição da cobertura de $B$. portulacoides ao trabalho de erosão do mar após as marés altas, equinociais ou de sizígia. Essa redução na cobertura desta espécie pela ação das ondas já foi verificada no Rio de Janeiro (Dansereau 1947; Magnanini 1954) e no Rio Grande do Sul (Pfadenhauer 1978). Bernardi et al. (1987) observaram que $B$. portulacoides dominava as dunas costeiras do sul do Brasil, sendo colonizadora na zona pioneira da praia (área desnuda da praia colonizada pelas plantas em períodos de calmarias), estando sujeita à destruição parcial ou total por ressacas.

Formação arbustiva de Palmae - nas restingas do sudeste brasileiro, a formação arbustiva de Palmae já foi reconhecida para os Estados do Rio de Janeiro (Araujo \& Henriques 1984; Sá 1992) e
Espírito Santo (Pereira 1990). Estudos quantitativos desta formação apontaram $A$. arenaria como dominante em Saquarema (RJ), onde esta espécie representa 55\% do VI total (Almeida \& Araujo 1997) e Guarapari (ES), nas restingas de Ulé e Carais, onde representa, respectivamente $39 \%$ e $72 \%$ do VI total (Cardoso 1995). Em comparação, neste estudo representa $56 \%$ do VI total. A dominância de $A$. arenaria na formação arbustiva de Palmae é, provavelmente, reflexo de sua capacidade de propagação através de caules subterrâneos (Tomlinson 1960; Rizzini 1979; Leite 1990) e da grande disponibilidade de frutos e sementes durante o ano (Leite 1990; Menezes 1996), além de sua resistência ao fogo (Araujo \& Peixoto 1977; Leite 1990; Cirne \& Scarano 1996; Menezes 1996).

$\mathrm{O}$ alto VI da forma de vida geófita rizomatosa na formação arbustiva de Palmae, na restinga de Marambaia, está diretamente relacionado com a dominância de $A$. arenaria, que apresenta tal característica, embora esta forma de vida tenha sido mais representada em número de espécies na formação psamófila-reptante. A predominância desta forma de vida em formações vegetais praianas está relacionada ao seu sucesso regenerativo através do estabelecimento de fragmentos vegetativos após as perturbações causadas pelas marés de tempestades (Crawford 1989; Packham \& Willis 1997).

Embora algumas espécies possam ser bastante comuns nesta formação (e.g. Schinus terebinthifolius, Cereus fernambucensis, Sophora tomentosa (Pereira 1990; Cardoso 1995; Almeida 1995; Menezes 1996), outras espécies se associam à $A$. arenaria, propiciando diferentes fisionomias da formação de Palmae ao longo do litoral do sudeste brasileiro, como Jacquinia brasiliensis e Campomanesia guazumaefolia em Setiba (ES - Pereira 1990), Diodea radula e Eugenia nitida em Saquarema (RJ Almeida \& Araujo 1997), Sebastiania glandulosa e Stigmaphyllon paralias em Ulé(ES - Cardoso 1995). Em Marambaia, A. arenaria compartilha a fisionomia da formação arbustiva de Palmae com Inga maritima e Manilkara subsericea.

Esta composição florística variada indica que não existe flora particular para essa formação, 
sendo, portanto, colonizada por espécies das formações vegetais mais próximas a ela e por espécies oportunistas. A influência destas formações, certamente, está relacionada com a posição topográfica na qual a formação arbustiva de Palmae se localiza. Nas restingas de Marambaia (Menezes 1996), Ulé e Carais (ES - Cardoso 1995) e Setiba (ES - Pereira 1990), esta formação encontra-se entre o mar e a crista do cordão externo. Já em Cabo Frio (RJ - Ulé 1901) e Saquarema (RJ - Almeida \& Araujo 1997) está presente na face do cordão externo voltado para o continente, próxima às formações vegetais interioranas, como a de Myrtaceae e de Clusia. Todas as nanofanerófitas encontradas na formação arbustiva de Palmae na restinga de Marambaia, por exemplo, também ocorrem na mata baixa que recobre as dunas, localizada após a estrada que limita essas formações. A proximidade da vegetação arbórea das dunas com a formação arbustiva de Palmae pode ser a razão para o acréscimo abrupto do número de espécies nas últimas parcelas desta formação.

A inexistência da formação halófila, comum em outras áreas litorâneas brasileiras, e a distribuição espacial azonal de B. portulacoides parecem estar diretamente ligadas a processos dinâmicos que determinam o perfil das praias. Dado a importância destes processos na erosão da zona costeira, é necessário realizar acompanhamento da cobertura vegetal durante vários ciclos de maré para se chegar a entendimento mais profundo sobre o histórico das formações vegetais nesta zona praiana. $\mathrm{O}$ alto grau de destruição da biota das restingas também exige agilidade nos estudos da diversidade florística que, embora demonstra certo grau de semelhança ac longo do litoral nesta zona praiana, especialmente quanto as espécies dominantes, também revela diversidade significativa de espécies menos abundantes.

\section{Referências bibliográficas}

Almeida, A. L. 1995. Fitossociologia das comunidades vegetais do cordão arenoso externo da Reserva Ecológica Estadual de Jacarepiá, Saquarema, RJ. Dissertação de Mestrado. Universidade Federal do Rio de Janeiro, Museu Nacional, Rio de Janeiro.
Almeida, A. L. \& Araujo, D. S. D. 1997. Comunidades vegetais do cordão arenoso externo da Reserva Ecológica Estadual de Jacarepiá, Saquarema, RJ. Oecologia Brasiliensis 3: 47-63.

Araujo, D. S. D. 1992. Vegetation types of sandy coastal plains of tropical Brazil: a first approximation. Pp. 337-347. In: U. Seeliger (Ed.), Coastal Plant Communities of Latin America. Academic Press, New York.

Araujo, D. S. D. \& Henriques, R. P. B. 1984. Análise florística das restingas do Estado do Rio de Janeiro. Pp.159-193. In: L. D. Lacerda, D. S. D. Araujo, R. Cerqueira \& B. Turcq (Orgs.), Restingas: Origem, Estrutura, Processos. CEUFF, Niterói.

Araujo, D. S. D. \& Oliveira, R. R. 1988. Reserva Biológica Estadual da Praia do Sul (Illha Grande, Estado do Rio de Janeiro): lista preliminar da flora. Acta Botanica Brasilica 1 (Supl.): 83-94.

Araujo, D. S. D. \& Peixoto, A. L. 1977. Renovação de uma comunidade vegetal de restinga após queimada. Pp. 117. In: Anais do XXVII Congresso Nacional de Botânica, Rio de Janeiro 1975. Academia Brasileira de Ciências, Rio de Janeiro.

Bastos, M. N. C. 1996. Caracterização das formações vegetais da Restinga da Princesa, Ilha de Algodoal, Pará. Tese de Doutorado. Universidade Federal do Pará, Museu Paraense Emílio Goeldi, Belém.

Bernardi, H.; Cordazzo, C. V. \& Costa, C. S. B. 1987. Efeito de ressacas sobre Blutaparon portulacoides (St. Hil.) Mears, nas dunas costeiras do sul do Brasil. Ciência e Cultura 39(5/6): 545-547.

Borges, H. V. 1990. Dinâmica sedimentar da restinga de Marambaia e Baía de Sepetiba. Dissertação de Mestrado. Universidade Federal do Rio de Janeiro, Rio de Janeiro.

Brower, J. E. \& Zar. J. H. 1977. Field and laboratory methods for general ecology. $2^{a}$ ed. Wm. C. Brown Co., Dubuque, Iowa.

Cardoso, M. S. R. 1995. Análise fitossociológica na formação Palmae de restinga no Parque Estadual Paulo César Vinha-Guarapari/ES. Monografia de Especialização. Universidade Federal do Espírito Santo, Vitória.

Cirne, P. \& Scarano, F. R. 1996. Rebrotamento após fogo de Andira legalis (Leguminosa) em restinga fluminense. Pp. 128-136. In: H. S. Miranda; C. H. Saito \& B. F. S. Dias (Eds.), Impactos de queimadas em áreas de cerrado $\mathrm{e}$ restinga. Universidade de Brasília, Brasilia.

Cordeiro, S. Z. 1998. Análise da cobertura vegetal em três áreas de topografia distinta na Praia do Peró, Cabo Frio, RJ. Dissertação de Mestrado. Universidade Federal do Rio de Janeiro, Museu Nacional, Rio de Janeiro.

Crawford, R. M. M. 1989. Studies in plant survival: ecological case histories of plant adaptation to adversity. Blackwell Scientific Publ., London.

Dansereau, P. 1947. Zonation et sucession sur la restinga de Rio de janeiro. I. Halosére. Revue Canadienne de Biologie 6(3): 448-477. 
Frangi, J. L. \& Lugo, A. E. 1998. A flood plain palm forest in the Luquillo Mountains of Puerto Rico five years after Hurricane Hugo. Biotropica 30(3): 339-348.

Garcia, G. J. \& Piedade, G. C. R. 1987. Topografia aplicada às ciências agrárias. $5^{\mathrm{a}} \mathrm{ed}$. Nobel, São Paulo.

Hay, J. D.; Henriques, R. P. B. \& Lima, D. M. 1981. Quantitative comparisons of dune and foredune vegetation in restinga ecosystems in the State of Rio de Janeiro. Revista Brasileira de Biologia 41(3): 655-62.

Henriques, R. P. B.; Meirelles, M. L. \& Hay, J. D. 1984. Ordenação e distribuição de espécies das comunidades vegetais na praia da restinga de Barra de Maricá, Rio de Janeiro. Revista Brasileira de Botânica 7: 27-36.

Henriques, R. P. B.; Araujo, D. S. D. \& Hay, J. D. 1986. Descrição e classificação dos tipos de vegetação da restinga de Carapebus, Rio de Janeiro. Revista Brasileira de Botânica 9: 173-189.

Hueck, K. 1955. Plantas e formações organogênicas das dunas do litoral paulista. Contribuição para a pesquisa fitossocilógica paulista. Secretaria da Agricultura, Instituto de Botânica, São Paulo.

Lacerda, L. D.; Araujo, D. S. D. \& Maciel, N. C. 1993. Dry coastal ecosystems of the tropical Brazilian coast. Pp.477493. In: E. van der Maarel (Ed.), Dry Coastal Ecosystems of the World. Elsevier, Amsterdam.

Lamego, A. R. 1946. Ciclo evolutivo das Lagunas Fluminenses. Boletim n. 116. Divisão de Geologia e Mineralogia, DNPM, Rio de Janeiro.

Leite, C. O. 1990. Biologia de reprodução de Allagoptera arenaria (Gomes) O. Kuntze (Diplotemium maritimum Mart.) - Palmae. Dissertação de Mestrado. Universidade Federal do Rio de Janeiro, Museu Nacional, Rio de Janeiro.

Magnanini, A. 1954. Contribuição ao estudo das zonas de vegetação da praia de Sernambetiba, DF. Arquivos do Serviço Florestal 8: 147-232.

Menezes, L. F. T. 1996. Caracterização de comunidades vegetais praianas da restinga de Marambaia-RJ. Dissertação de Mestrado. Universidade Federal Rural do Rio de Janeiro, Seropédica.

Menezes, L. F. T.; Araujo, D. S. D. \& Goes, M. H. B. 1998. Marambaia: a última restinga carioca preservada. Ciência Hoje 136(23): 28-37.

Menezes-Silva, S. 1998. As formações vegetais da planície litorânea da Ilha do Mel, Paraná, Brasil: composição florística e principais características estruturais. Tese de Doutorado. Universidade Estadual de Campinas, Campinas.

Montagnini, F.; Eibl, B.; Szczipanski, L. \& Ríos, R. 1998. Tree regeneration and species diversity following conventional and uniform spacing methods of selective cutting in a subtropical humid forest reserve. Biotropica 30(3): 349-361.

Mueller-Dombois, D. \& Ellenberg, H. 1974. Aims and methods of vegetation ecology. John Wiley \& Sons, New York.

Oliveira-Filho, A. T. 1993. Gradient analysis of an area of coastal vegetation in the state of Paraiba, northeastern Brazil. Edinburgh Journal of Botany 50(2): 217-236.
Oliveira-Filho, A. T.; Curi, N.; Vilela, E. A. \& Carvalho, D. A. 1998. Effects of canopy gaps, topography and soils on the distribution of woody species in a central Brazilian deciduous dry forest. Biotropica 30(3): 362-375.

Packham, J. R. \& Willis, A. J. 1997. Ecology of dunes, salt marsh and shingle. Chapman \& Hall, London.

Pereira, O. J. 1990. Caracterização fitofisionomica da restinga de Setiba, Guarapari, E.S. Pp. vol.3: 207-219. In: Anais do II Simpósio de Ecossistemas da Costa Sul e Sudeste Brasileira: estrutura, função e manejo. ACIESP, Águas de Lindóia, São Paulo.

Pereira, O. J.; Thomaz, L. D. \& Araujo, D. S. D. 1992. Fitossociologia da vegetação de antedunas da restinga de Setiba/Guarapari e em Interlagos/Vila Velha, ES. Boletim do Museu de Biologia Mello Leitão (N. Ser.) 1: 65-75.

Pfadenhauer, J 1978. Contribuição ao conhecimento da vegetação e de suas condições de crescimento nas dunas costeiras do Rio Grande do Sul. Brasil. Revista Brasileira de Biologia 38: 827-836.

Pfadenhauer, J. 1993. Dry coastal ecosystems of temperate Atlantic South America. Pp. 495-500. In: E. van der Maarel (Ed.), Dry Coastal Ecosystems of the World. Elsevier, Amsterdam.

Rizzini, C. T. 1979. Tratado de fitogeografia do Brasil: aspectos sociológicos e florísticos. vol. 2. HUCITECEDUSP, São Paulo.

Roncarati, H. \& Barrocas, S. L. S. 1978. Estudo preliminar dos sedimentos recentes superficiais da Baía de Sepetiba, Município do Rio de Janeiro - Itaguaí e Mangaratiba-RJ. CENPES/Petrobrás, Rio de Janeiro.

Sá, C. F. C. 1992. A vegetação da restinga de Ipitangas, Reserva Ecológica Estadual de Jacarepiá, Saquarema(RJ). Arquivos do Jardim Botânico do Rio de Janeiro 31: 87-102.

Seeliger, U. 1992. Coastal foredunes of southern Brazil: physiography, habitats and vegetation. Pp. 367-381. In: U. Seeliger (Ed.), Coastal Plant Communities of Latin America. Academic Press, New York.

Suguio, K. \& Martin, L. 1990. Geomorfologia das restingas. Pp. vol. 3: 185-205. In: Anais do II Simpósio de Ecossistemas da Costa Sul e Sudeste Brasileira: estrutura, função e manejo. ACIESP, Águas de Lindóia, São Paulo.

Thomaz, L. D. 1991. Distribuição e diversidade de espécies na vegetação hálófila-psamófila no litoral do Espírito Santo. Dissertação de Mestrado. Universidade Estadual Paulista, Rio Claro.

Thomaz, L. D. \& Monteiro, R. 1993. Distribuição das espécies na comunidade halófila-psamófila ao longo do litoral do Estado do Espírito Santo. Arquivos de Biologia e Tecnologia 36 (2): 375-399.

Tomlinson, P. B. 1960. Essays on the morphology of palms. II. The early growth of the palm. Principes 4 (4): 140-143.

Waechter, J. L. 1990. Comunidades vegetais de restingas do Rio Grande do Sul. Pp. vol. 3: 228-248. In: Anais do II Simpósio de Ecossistemas da Costa Sul e Sudeste Brasileira: estrutura, função e manejo. ACIESP, Águas de Lindóia, São Paulo. 